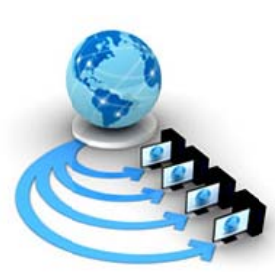

Volume 9, No. 1, January-February 2018

International Journal of Advanced Research in Computer Science

SURVEY REPORT

\title{
5G CELLULAR NETWORK'S DEVICE TO DEVICE COMMUNICATION: A STATE-OF-THE-ART-SURVEY
}

\author{
Surinder Singh Khurana \\ Ph.D. Scholar \\ Punjab Technical University, India
}

\author{
Manju Bala \\ Professor \& Head, \\ Computer Science and Engineering \\ Khalsa College of Engineering \& Technology, India
}

\author{
Sukhvinder Singh Bamber \\ Assistant Professor \\ Panjab University SSG Regional Centre, India
}

\begin{abstract}
Device to Device communication is a must required technology to support rapidly growing internet enabled devices. These devices are dependent on cellular technologies to access the data services. Devices in the proximity use Device-to-Device Communication to directly communicate, hence reduce the burden on the base station. It allows for communication between devices without the use of any core network infrastructure but within the licensed band. It is becoming an increasingly popular concept especially when used in context of 5G cellular networks as it offers many advantages like lowering the communication cost, increasing the link reliability, gain in spectral efficiency and system capacity. This paper presents a state of the art survey in the field of D2D communication. Furthermore we also discussed open research issues in the field.
\end{abstract}

Keywords: Device to Device Communication; 5G; Data Offloading; Inband Communication; Outband Communication

\section{INTRODUCTION}

The demand of the mobile devices is growing with a very remarkable speed. Around 90\% of the world's population will be connected to mobile phone by 2020[1]. Furthermore the usage pattern of these devices is also varying rapidly. Now more attention is towards the Internet based services. Electronic equipments other than mobile phones are also joining the smart world connected through Internet. All these devices are contributing to huge amount of data generation and its communication. To provide a better quality to these devices network services are need to be upgraded. The Fourth Generation(4G) Wireless Networks are being deployed in various countries and are able to handle current requirements. However the technologies used by these networks are capable of handling future requirements as more than 50 billion devices are predicted to join Internet by 2020 [2]. The future applications demand reliable communication at very high speed. For example driverless cars need data packets to arrive quickly so that the decision can be made and maps can be updated. The goal of fifth generation wireless networks (5G) is to enable communication between this gigantic set of devices with much faster speed. Furthermore, 5G will also support high quality video streaming, TV, Video on demand services. As per the researcher's observation a mobile user use the services from inside a building for around $80 \%$ time and from outside the buildings around 20\% [3]. Even the conventional 5G cellular technology will not be able to support these ever rising demands of the higher data rates. So a new technology has emerged which is called the Device-to-Device (D2D) technology [4]. It is a technology that aims to provide direct communication between wireless devices without the use of the core cellular network infrastructure.

In the traditional cellular network that we have been using till date all transmission was carried out by the fixed infrastructure based base stations (BS). No two devices could directly connect with each other without the use of the base station. It is called the fixed terminal relaying[4]. D2D is a form of cooperative communication[5] in which the devices cooperate with each other to relay information from one device to another via intermediate devices that act as transmission relaying devices for each other. This is called device relaying. The term device can be used for any wireless node that has cellular connectivity i.e. a tablet, a laptop or even a mobile phone. With device relaying we get a huge and extensive adhoc network that looks like a mesh of heterogeneous devices. In D2D technology the direct communication between the two nodes will be via the licensed cellular bandwidth. It is possible with mainly two ways: firstly with limited role of a base station or secondly even without any role of base station involved in the communication between two devices.

D2D communication has not particularly been implemented or researched from $1 \mathrm{G}$ to $4 \mathrm{G}$ cellular systems. This is because most of the local operators didn't find it a tool of much importance until recently. Now when the context-aware services and location based applications are increasing and these require communication between neighboring devices, the interest has been shifted towards implementing the D2D communication with the $5 \mathrm{G}$ cellular systems. This may be due to multifold benefits of D2D communication. These benefits include:

1) Lower cost: The rate of communication between devices is minimized as the communication does not actively involve the base station at each step of communication in a D2D communication.

2) Gain in link reliability: Even if a link between a pair of devices is broken, we can relay information between devices using the other intermediate devices. So the links in D2D communication have increased the link reliability better than the traditional cellular systems. 
3) Spectral efficiency: Information rate that can be exchanged over a specific bandwidth between devices in D2D communication is maximized. Hence it has efficient bandwidth utilization.

4) Gain in system capacity: As the number of devices operating in a particular cell can be maximized to a higher value than in a traditional system, the system capacity of D2D communication networks is more than the traditional cellular networks.

5) Increased transmission range: The devices that are far away from one another's transmission range can also communicate with each other via the intermediate devices.

\section{APPLICATION AREAS OF D2D COMMUNICATION}

D2D communication would successfully help in reducing the cost of communication between two devices. Another application of D2D communication is the offering of mobile cloud computing services via D2D communication. With D2D communication one device can use the computing power, storage space, spectrum, applications or even the infrastructure of its nearby devices. The daily load on the local area network can also be reduced to a great extent when the direct transmission relays take place via D2D communication. D2D communication plays a vital role after natural disasters like an earthquake. An earthquake or even a flood may damage the fixed infrastructure based transmission station and hence it disrupts all communication in the area affected by the natural calamity. In such cases of emergency D2D communication can be used to provide communication facilities to nodes without requiring any infrastructure such as Base Stations. In this way nodes in proximity can communicate with each other. Furthermore, the communication between nodes that are far away from each other may be supported by using multichip communication. In this case the message is communicated through multiple intermediately nodes which act as relay nodes. Multicasting applications can also make efficient use of this D2D technology. Newer areas of application of D2D communication involve vehicle-to-vehicle communication which is another aspect of Internet-of-Things (IOT). Vehicleto-vehicle communication involves the interaction of vehicles moving on a road and which tell each other about the amount of traffic they witness, the collisions to avoid and the optimal less traffic routes to follow to reach a particular destination. Hence, they also help avoid accidents and collisions with each other.

\section{DeVice TO DEVICE COMMUNiCATION STRUCTURAL DESIGN}

To support D2D communication and exploits its full strength, few changes are required to existing cellular network structure. To support D2D communication, a cellular network model will involve two tiers namely, the macro cell tier and the device tier. Hence, it will be a two tier cellular network structure [4] with some improvements to the traditionally used cellular network architecture. The macro cell tier will be responsible for all interactions between the device and the base station (BS). Whereas the device tier will be responsible for all interactions between the devices only means for device-todevice communication. If a device is connected to the BS we shall say that the device is involved in the tier called macro cell. If case a device is connected to other device and is transmitting or relaying information via intermediate devices, we shall say that the device is involved in the device tier. To effectively allocate the resources in D2D communication sectored antennas can be deployed at base station.

The first tier i.e macro cell is almost similar to existing cellular system and provides a fixed infrastructure. The main difference between this structure and is due to the introduction of device tier in the structure. Furthermore, the functioning of the device tier varies according to the level of involvement of Base Station and other entities controlled by cellular service provider, in deciding the way devices communicate the through D2D Communication[4]. These variations are mentioned below:

"Device relaying with operator controlled link establishment (DR-OC)"[4]: This type of functionality enables the base station to communicate with the nodes that are out of its coverage area. Such type of nodes communicates with the base station through other intermediate nodes which fall under the coverage area of the base station. These intermediate nodes communicate just as relay nodes. This functionality extends the communication model and provides a way to base station to communicate with out of coverage area nodes and vice versa. At the one hand in extends the capability by supporting long distance communication and on other hand it also introduces various security threats as intermediate nodes can perform various active and passive attacks.

"Direct D2D communication with operator controlled link establishment (DC-OC)"[4]

This variation of device tier allows the device to communicate with each other. The data from one device to other is directly communicated to other device without involving the base station. However, the topology is decided with the decision made and conveyed by the base station. These decisions includes about the link establishment between various devices that are going to communicate directly.

"Device relaying with device controlled link establishment (DR-DC)',[4]:

Here in this mode the source and destination devices communicate with each other through the intermediate nodes. The base station is not involved in this communication in any role. The topology is created through distributed decision, where all the decisions about link establishments are taken by the involved devices. This will reduce the burden of base station.

"Direct D2D communication with device controlled link establishment (DC-DC)”[4]:

Here in this mode the source device communicates with destination device without involving any relaying device or base station. The topology is also created by the devices without the interference of the base station.

During the link establishment radio channels are allocated for required D2D communications. Hence, the D2D communication can take place via the following modes:

Silent mode: In this mode no transmission of data takes place by D2D devices because of non-availability of resources. Hence, the D2D devices are said to be silent.

Dedicated mode: In this mode the D2D devices have been allocated some specific resources for their usage in the direct links between the devices.

Reuse mode: This mode enables the $\mathrm{D} 2 \mathrm{D}$ devices to make use of the free uplink and downlink channels of the cellular users.

Cellular mode: In this type of conventional communication D2D data and information is transferred via the eNBs. 


\section{Classification OF D2D Communication}

D2D communication can be classified into two categories[6]: Inband D2D communication and Outband D2D communication. Its types and subtypes are mentioned in figure2.

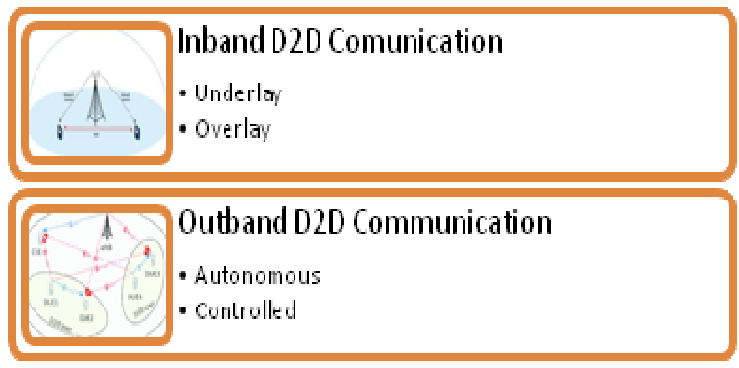

Figure-2: Types of D2D Communication

In reference to D2D communication, Inband and Outband communication is defined based on the communication channels used for the purpose. In case of Inband D2D communication cellular channels are used for the D2D communication. Hence the base station to device communication and the direct communication between the device require the allocation of resources from the same cellular bandwidth and both of these devices contend for the same channels. This lead to the requirement of efficient channel allocation mechanism to reduce the interference and increase the channel utilization. On the other side, in case of Outband communication devices will use different wireless channels such as channels used in wifi-direct[7], Bluetooth[8], zigbee[9], for directly communicating with other devices in the proximity. This approach will increase the network capacity to a higher extent as compared to Inband D2D communication. Other difference between Inband and Outband D2D communication is that the licensed bandwidth spectrum is used for Inband D2D communication but the Outband communication operates over unlicensed spectrum.

Many approaches have been proposed for D2D communication based on this mechanism. The main motive of the research in this direction is to reduce the interference between cellular and D2D communications. In case of overlay communication the network channels are dedicatedly allocated for cellular as well D2D communications. Although the overlay communication reduce the interference, but it is not able achieve spectral efficiency as underlay communication can achieve.

Main challenge in the area is efficient allocation of the radio channels with interference management. Various approaches were introduced to handle these issues. One category of the approaches[10],[11],[12],[13],[14] are based on the assumption that base station is aware of cellular and D2D communication taking place at the moment. On the other side approaches[15] [16] have also been proposed which assumes that the base station and device nodes are not aware about cellular and D2D communication taking place at that moment. An approach that exploits the fact about low utilization of uplink channels [17] was proposed for $\mathrm{d} 2 \mathrm{~d}$ inband communication. The author suggested to use the unutilized spectrum from uplink spectrum for D2D communication. However such kind of approach only offers limited extendibility. In another such approach [18], the authors proposed to communicate $\mathrm{d} 2 \mathrm{~d}$ communications during uplink frame. Using such approaches only base station is exposed to interference. To maximize the throughput and user satisfaction a technique was introduced by Chen et al. [19]. They used non-orthogonal sharing mode. For further improvements, the authors in Zheng et al. [20] proposed the use of allocating timeslots to D2D pairs. The timeslots are allocated adaptively. The work is based on the improved proportional fairness algorithm. A two stage resource allocation scheme[21] has also been proposed to achieve high resource utilization. It uses heuristic greedy resource strategy and Langrangian dual method based power allocation algorithm for subcarrier assignment. As the main issue with overlay communication is to improve spectral utilization Yang \& Kuo[22] proposed an efficient approach to allocate network resources to cellular and D2D communications. Their approach is based on finding minimum overlay partition. To find so they defined an algorithm called largest aggregated interference first algorithm (LIFA) which works based on greedy methodology.

Outband D2D Communications: In outband communication concept of data offloading is used. To reduce the burden on cellular channels D2D communications will be shifted to non cellular networks. These networks may be Bluetooth, Wifi, WiMax etc. Outband D2D communication can be either network controlled or device controlled(autonomous). In network controlled the base station form the group and inform the nodes about the medium through which these nodes will communicate. As multiple heterogeneous networks are being used in outband communication the spectral availability is increased by multifold.

Many schemes have been proposed to resolve various issues involved in outband communication. In [23], Hematian et. al. studied the use of wi-fi direct to offload the data traffic from cellular communications. Few other schemes are also available which works on the same idea to offload the cellular data to wifi networks. The data related to nodes in a close proximity is suggested to be communicated via wi-fi/ wi-fi direct network. For the purpose it is suggested to form the clusters.

In [24], authors proposed use of Bluetooth technology to initially form the clusters and to use wi-fi network for data offloading. In [25], Asadi et. al. presented D2D Opportunistic Relay with QoS Enforcement (DORE) for improving channel capacity with maintaining Quality of servce in terms of throughput.

Cluster optimization to reduce energy requirements and improve quality of services is still an open research issue for outband communication.

\section{SIMULATORS FOR D2D COMMUNICATIONS}

There are only few simulators which supports the D2D communication with cellular communication. These simulators tools are:

LENA[26]: It's a NS3 module which simulates LTE-A. Other modules such as Wifi, available in NS3 enables to simulate outband D2D communication. The module is coded in $\mathrm{C}++$.

SimuLTE [27]: It's a Omnet++ module developed for developed to simulate LTE and LTE-A networks. It supports lot of features of Physical and MAC layer. The code if the module is written in $\mathrm{C}++$.

LTE-Sim [28]: LTE-Sim is standalone simulator written in $\mathrm{C}++$. Its event driven simulator which supports both uplink and downlink simulations. This tool needs to be extended to support D2D communications. 
Vienna-LTE [29]: Vienna Simulator is written in Matlab. There are various variants of this simulator which includes: Vienna link level simulator and Vienna system level simulator. This tool also needs to be extended to support D2D communications.

NetSim[30]: NetSim is proprietary tool. The support for LTE D2D simulation is provided in NetSim v10.

Except NetSim all the tools are free open source simulators. However very limited functionalities are implemented in these tools to support D2D communications.

\section{CHALLENGES IN D2D COMMUNICATION}

The main challenge posed in D2D communication is security point of view. In D2D communication the relaying of information between any two devices occurs via intermediate devices acting as the relaying devices. It is in stark contrast to the traditional cellular system in which the relaying part was the work of the base station only. Here, in D2D the user data while routing goes through relaying devices and hence the security of the data can be compromised easily if efficient security mechanisms are not implemented. A solution to this security threat is to use the closed access technique. In this technique all the devices that are currently operating in the device tier of the D2D communication network need to maintain a list of its trusted devices. The device will make any other device a trusted device only when that device has authenticated itself. These trusted devices mainly belong to a particular workplace or neighborhood. So these groups of trusted devices should only be allowed to communicate with each other directly via the device tier system. If any other non trusted device needs to communicate to any other device then it needs to do via the macro-cell tier system only and not via the device tier. Hence this approach limits the communication.

Whereas in the open access technique any device can access any other device and can make it relay its information. It has no strict rules and regulations like the closed access technique. This is more dangerous as far as security is concerned as there is no control over the possible threats, attacks as well as security breach of one device by another device during the relaying of its data.

As D2D communication allows communicating the data through relay nodes/cluster heads all security issues as applicable to Mobile Ad-hoc Networks are also applicable to D2D communications. These attacks includes: Gray Hole, Black Hole, Eavesdropping, Man in the middle, session hijacking etc. In addition few new type of attacks may also be applicable. We identified one such attack called SelectiveSlowdown-Attack. When the device offloads the communication to non cellular network such as wifi, usually packets will be communicated through cluster head which will be selected among one of the mobile devices. The depletion of energy of the node is directly proportional to the speed at which the node is communicating. To save the battery power a selected cluster head node can treat in selfish manner and will forward the data belonging to other nodes at lower rates.

Considering the types of device tier D2D communication available we can see that the DR-OC and DC-OC techniques involve the base station services for resource allocation and call setup mechanisms. In DR-DC and DC-DC techniques we have no involvement of the base station or any central agency to monitor the device-to-device communication. Hence the D2D communication in DR-OC and DC-OC is more secure than the D2D communication via DR-DC and DC-DC techniques. In DR-DC and DC-DC there are no means of centralized relaying and supervision making it tough to maintain secure control in these areas.

Efficient cluster formation is also a research issue for outband D2D communication. The motive of cluster formation is to reduce interference, increase capacity of network to handle D2D pairs, and to increase network lifetime.

Another major challenge is the problem of pricing for D2D technology. This technology does involve the operator in some cases like DR-OC and DC-OC. But the problem of fixing the price remains on the operator side as well as the customer side. The customers should pay for what services remains a question to be pondered over. The operator can create a secure and healthy environment for the devices involved in D2D communication and hence can be paid by the customers for this reason.

Handover management is another such open issue in D2D communication. When a device will move from the area controlled by one base station to another base station controlled area it has to follow certain handover techniques so as to avoid call drops etc.

In context of D2D communication particularly in the DR-DC and DC-DC it will be a challenging task as there is no base station's involvement involved. So if a device which is under the DR-DC or DC-DC communication changes its base station cell area ie. it moves to another cell and there in the new cell it starts a DR-OC or DC-OC communication it will be problematic as the old base station will not know that the device has left its range and has joined a new cell base station. So handover needs to be addressed in this context.

Furthermore performance analysis of mixed mode (using both inband and inband modes) D2D communication is need to carried out in future. The use of combination of inband and outband mode will improve the spectral efficiency.

\section{REFERENCES}

[1] Ericsson Mobility Report: On the Pulse of the Networked Society, Ericsson, 2015. Available at: http://www.ericsson.com/res/docs/ 2015/ericsson-mobilityreport-june-2015.pdf.

[2] D. Evans, "The Internet of Things How the Next Evolution of the Internet Is Changing Everything”, Cisco White Paper. http://www.cisco.com/c/dam/en_us/about/ac79/docs/innov/I oT_IBSG_0411FINAL.pdf. Accessed on 10 Jan 2017.

[3] V. Chandrasekhar, J. G. Andrews, and A. Gatherer, "Femtocell networks: A survey," IEEE Commun. Mag., vol. 46, no. 9, pp. 59-67, Sep. 2008.

[4] M. N. Tehrani , M. Uysal, and H. Yanikomeroglu. "Deviceto-device communication in 5G cellular networks: challenges, solutions, and future directions." IEEE Communications Magazine 52.5 (2014): 86-92.

[5] Cao, Yang, Tao Jiang, and Chonggang Wang. "Cooperative device-to-device communications in cellular networks." IEEE Wireless Communications 22.3 (2015): 124-129.

[6] A. Asadi, W. Qing, and M. Vincenzo. "A survey on deviceto-device communication in cellular networks." IEEE Communications Surveys \& Tutorials 16.4 (2014): 18011819.

[7] W. ALLIANCE, "Wi-Fi Peer-to-Peer (P2P) Specification v1. 1, "WI-FI ALLIANCE SPECIFICATION, vol. 1, pp. 1-159, 2010

[8] Specifications, Bluetooth, "Bluetooth specification version 1.1,” Available HTTP: http://www.bluetooth.com, 2001.

Zigbee Alliance, “Zigbee specification,” Document 053474r06, Version, vol. 1, 2006. 
[9] X. Xiao, X. Tao, and J. Lu, “A QoS-aware power optimizatiOn scheme in OFDMA systems with integrated device-to-device ( D2D) communications,” in Proceedings of IEEE VTC-Fall, 2011, pp. 1-5.

[10] D. Feng, L. Lu, Y. Yuan-Wu, G. Y. Li, G. Feng, and S. Li, "De vice-to- device communications underlaying cellular networks," IEEE Trans action

[11] L. Su, Y. Ji, P. Wang, and F. Liu, "Resource allocation using particle swarm optimization for D2D communication underlay of cellular networks," in Proceedings of IEEE WCNC , 2013, pp. 129-133.

[12] C. H. Yu, O. Tirkkonen, K. Doppler, and C. Ribeiro, "Power optimization of device-to-device communication underlaying cellular communication,” in Proceedings of IEEE ICC , 2009, pp. 1-5.

[13] T. Han, R. Yin, Y. Xu, and G. Yu, "Uplink channel reusing selection optimization for device-to-device communication underlaying cellular networks," in Proceedings of IEEE PIMRC , 2012, pp. 559-564.

[14] C. H. Yu, K. Doppler, C. Ribeiro, and O. Tirkkonen, "Performance impact of fading interference to device-todevice communication underlaying cellular networks," in Proceedings of IEEE PIMRC , 2009, pp. 858-862.

[15] Q. Wang and B. Rengarajan, "Recouping opportunistic gain in dense base station layouts through energy-aware user cooperation ," in Proceedings of IEEE WoWMoM , 2013, pp. 1-9.

[16] K. Huang, Y. Chen, B. Chen, X. Yang, and V. Lau , "Overlaid cellular and mobile ad hoc networks." Communication Systems, 2008. ICCS 2008. 11th IEEE Singapore International Conference on. IEEE, 2008.

[17] B. Kaufman, and A. Behnaam. "Cellular networks with an overlaid device to device network." Signals, Systems and Computers, 2008 42nd Asilomar Conference on. IEEE, 2008.

[18] X. Chen, B. Proulx, X. Gong, and J. Zhang. "Exploiting social ties for cooperative D2D communications: A mobile social networking case." IEEE/ACM Transactions on Networking 23.5 (2015): 1471-1484.

[19] J. Zheng, B. Chen, and Y. Zhang. "An adaptive time division scheduling based resource allocation algorithm for D2D communication underlaying cellular networks." Global
Communications Conference (GLOBECOM), 2015 IEEE. IEEE, 2015.

[20] Y. Zhang, F. Li, M. Al-Qaness, and X. Luan, "A Resource Allocation Scheme for Multi-D2D Communications Underlying Cellular Networks with Multi-Subcarrier Reusing." Applied Sciences 7.2 (2017): 148.

[21] Z. Yang, and K. Yaw-Wen, "Efficient Resource Allocation Algorithm for Overlay D2D Communication." Computer Networks (2017).

[22] A. Hematian, W. Yu, C. Lu, D. Griffith, and N. Golmie, "A Clustering-Based Device-to-Device Communication to Support Diverse Applications." Proceedings of the International Conference on Research in Adaptive and Convergent Systems. ACM, 2016.

[23] Z. Lin, Z. Gao, L. Huang, C. Chen, H. Chao, "Hybrid architecture performance analysis for device-to-device communication in 5G cellular network." Mobile Networks and Applications 20.6 (2015): 713-724.

[24] A. Asadi, M. Vincenzo, and R. Gupta. "DORE: An Experimental Framework to Enable Outband D2D Relay in Cellular Networks." arXiv preprint arXiv:1706.01348 (2017).

[25] N. Baldo. "The ns-3 LTE module by the LENA project", 2011, Avialable at: https://www2.nsnam.org/tutorials/tutorials/consortium13/ltetutorial.pdf. Accessed on 12 April 2017.

[26] A. Virdis,S. Giovanni, and N. Giovanni, "Simulating LTE/LTE-Advanced Networks with SimuLTE." Simulation and Modeling Methodologies, Technologies and Applications. Springer International Publishing, 2015. 83105.

[27] G. Piro, L. A. Grieco, G. Boggia, F. Capozzi, P. Camard, "Simulating LTE cellular systems: An open-source framework." IEEE transactions on vehicular technology 60.2 (2011): 498-513.

[28] C. Mehlführer, et al. "The Vienna LTE simulators-Enabling reproducibility in wireless communications research." EURASIP Journal on Advances in Signal Processing 2011.1 (2011): 29.

[29] NetSim V10 Features, Available at: http://www.tetcos.com/new-releases.html, Accessed on: 15 June 